\title{
VECTor: A Preclinical Imaging System for Simultaneous Submillimeter SPECT and PET
}

\author{
Marlies C. Goorden*1, Frans van der Have*1,2, Rob Kreuger ${ }^{1}$, Ruud M. Ramakers ${ }^{1,2}$, Brendan Vastenhouw ${ }^{1,2}$, \\ J. Peter H. Burbach ${ }^{3}$, Jan Booij ${ }^{4}$, Carla F.M. Molthoff ${ }^{5}$, and Freek J. Beekman ${ }^{1,2}$ \\ ${ }^{I}$ Delft University of Technology, Delft, The Netherlands; ${ }^{2}$ MILabs, Utrecht, The Netherlands; ${ }^{3}$ Rudolf Magnus Institute of \\ Neuroscience, University Medical Center Utrecht, Utrecht, The Netherlands; ${ }^{4}$ Department of Nuclear Medicine, Academic Medical \\ Center, University of Amsterdam, Amsterdam, The Netherlands; and ${ }^{5}$ Department of Nuclear Medicine and PET Research, VU \\ University Medical Center, Amsterdam, The Netherlands
}

Today, PET and SPECT tracers cannot be imaged simultaneously at high resolutions but require 2 separate imaging systems. This paper introduces a Versatile Emission Computed Tomography system (VECTor) for radionuclides that enables simultaneous submillimeter imaging of single-photon and positron-emitting radiolabeled molecules. Methods: $\gamma$-photons produced both by electron-positron annihilation and by single-photon emitters are projected onto the same detectors by means of a novel cylindric high-energy collimator containing 162 narrow pinholes that are grouped in clusters. This collimator is placed in an existing SPECT system (U-SPECT-II) with 3 large-field-of-view $\gamma$-detectors. From the acquired projections, PET and SPECT images are obtained using statistical image reconstruction that corrects for energy-dependent system blurring. Results: For PET tracers, the tomographic resolution obtained with a Jaszczak hot rod phantom was less than $0.8 \mathrm{~mm}$, and $0.5-\mathrm{mm}$ resolution images of SPECT tracers were acquired simultaneously. SPECT images were barely degraded by the simultaneous presence of a PET tracer, even when the activity concentration of the PET tracer exceeded that of the SPECT tracer by up to a factor of 2.5. Furthermore, we simultaneously acquired fully registered 3- and 4-dimensional multiple functional images from living mice that, in the past, could be obtained only sequentially. Conclusion: High-resolution complementary information about tissue function contained in SPECT and PET tracer distributions can now be obtained simultaneously using a fully integrated imaging device. These combined unique capabilities pave the way for new perspectives in imaging the biologic systems of rodents.

Key Words: pinhole; SPECT; PET; small-animal; molecular imaging; neuroimaging; cardiac imaging

J Nucl Med 2013; 54:306-312

DOI: 10.2967/jnumed.112.109538

Received Jun. 1, 2012; revision accepted Sep. 5, 2012.

For correspondence or reprints contact: Freek J. Beekman, Delft University

of Technology, Mekelweg 15, Delft 2629 JB, The Netherlands.

E-mail: f.j.beekman@tudelft.nl

Published online Oct. 17, 2012

${ }^{*}$ Contributed equally to this work.

COPYRIGHT @ 2013 by the Society of Nuclear Medicine and Molecular Imaging, Inc. maging the distributions of radiolabeled molecules in small animals facilitates in vivo quantitative assessments of molecular mechanisms and the development of new pharmaceuticals and diagnostic tracers $(1,2)$. In molecular imaging, SPECT and PET are key modalities, offering the ability to track and quantify radiolabeled biomarkers (radiotracers or radiopharmaceuticals) with detection sensitivity below the nano- or even picomolar range. Current small-animal SPECT scanners can be used to image one or multiple SPECT tracers in a single scan with image resolutions that have improved enormously over the last decade (3-7), well below $0.5 \mathrm{~mm}$ in 3 dimensions $(8,9)$. In contrast, high-end commercial smallanimal PET attains image resolutions of approximately $1 \mathrm{~mm}$ (10-15) and cannot distinguish between simultaneously injected tracers, because positron emission from different tracers results in $\gamma$-photon pairs with equal energy $(511 \mathrm{keV})$. Recently, it was also shown that fast dynamic (total body) imaging is possible with small-animal SPECT based on stationary detectors $(16,17)$. This ability makes SPECT, besides PET, an important tool in, for example, kinetic modeling.

Using complementary sets of radiolabeled molecules, PET and SPECT make it possible to visualize and quantify different aspects of biologic function. However, today's scanners cannot take advantage of the entire complement of available radiotracers because, until now, these 2 modalities could not be combined into a single integrated system capable of imaging both classes of tracers simultaneously at high resolutions. Here, we present the first truly integrated submillimeter SPECT/PET platform that is designed to use a single set of detectors and a single user interface. In contrast to 2 standalone systems or 2 separate systems attached to one another in a series, simultaneous SPECT/PET acquisition provides perfectly aligned images in space and time of different tissue functions that can therefore be directly correlated. Furthermore, such a fully integrated SPECT/PET scanner not only saves costs, imaging time, training time, and floor space but also enables the imaging of PET and SPECT probes with a single dose of anesthesia.

Traditionally, PET has been based on a ring detector using dedicated electronics for the coincidence detection of 
pairs of annihilation photons. In contrast, SPECT systems use collimators to detect single $\gamma$-photons emitted by SPECT tracers. In several studies over the last decade, it has been shown that dedicated SPECT provides higher-resolution images of mice than dedicated PET does, because several image-degrading effects that play a role in small-animal PET are not present or are dramatically reduced with pinhole imaging (18). Therefore, we developed a novel collimation technique to enable separate imaging of the 2 high-energy $\gamma$-photons emitted by PET tracers simultaneously with photons emitted by SPECT tracers. This technique exploits clusters of pinholes (18-20), where each cluster samples the same field of view as a single traditional pinhole (Fig. 1A). Pinhole edge penetration, which would be a major problem were standard SPECT collimators to be used for high-energy annihilation photons, is dramatically reduced because each of the pinholes in a cluster has a smaller opening angle, thereby increasing the path length that a photon has to travel to penetrate the pinhole at a certain distance from the edge of the hole.

Here we present and apply the experimental realization of submillimeter simultaneous SPECT/PET based on clustered pinholes. This prototype Versatile Emission Computed Tomography system (dubbed VECTor, and available through MILabs) is based on a specially designed cylindric collimator containing 48 clusters of 4 pinholes each (Fig. 1B) that was placed into an existing SPECT system with integrated CT (U-SPECT-II/CT [MILabs], Fig. 1C). Similar to the standard multipinhole SPECT collimators of this system, the clustered-pinhole collimator applies a focusing geometry; all pinhole clusters focus on a central scan volume. This design allows the count yield from a specific organ or a tumor to be increased (21) but also allows imaging of larger regionsup to (dynamic) whole-body mouse scans $(17,22)$. We present a quantitative performance evaluation of VECTor using phantom data and show the results of simultaneously acquired

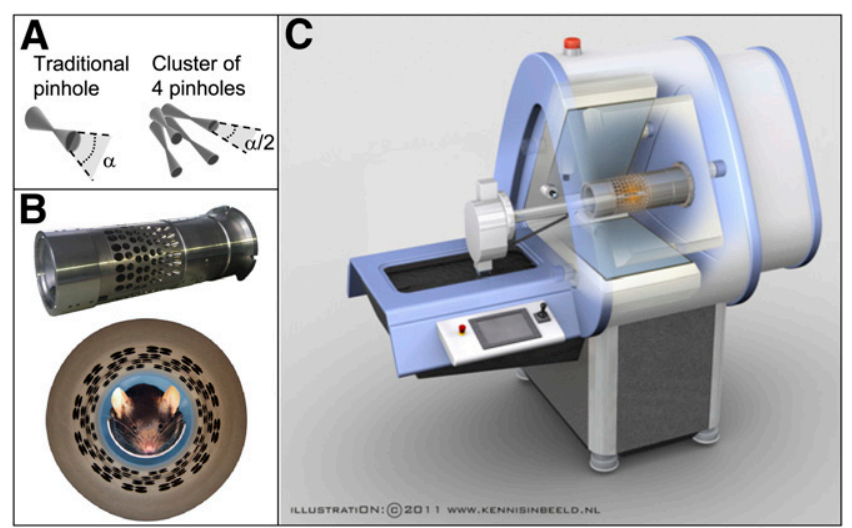

FIGURE 1. Integration of clustered-pinhole collimator into existing SPECT/CT platform. (A) Traditional pinhole with opening angle $\alpha$ and cluster of 4 pinholes with approximately same field of view and opening angle $\alpha / 2$. (B) Clustered-pinhole collimator optimized for imaging SPECT and PET tracers, into which mouse is placed. (C) Collimator mounted in SPECT/CT system. multiple functional data obtained with positron and singlephoton emitters in mice.

\section{MATERIALS AND METHODS}

\section{Pinhole Geometry and System Description}

The collimator that enables imaging of single-photon and positron emitters was integrated in the U-SPECT-II/CT system (8), which uses 3 large-field-of-view $\gamma$-detectors. The imaging platform has been dubbed VECTor. The tungsten collimator (shown in Fig. 1B) contains 48 clusters of four 0.7-mm-diameter pinholes (illustrated in Fig. 1A) placed in 4 rings. The pinholes in the inner 2 rings of the collimator have opening angles of $18^{\circ}$, whereas in the outer rings opening angles of $16^{\circ}$ were chosen in order to achieve a more uniform coverage of the detectors with pinhole projections. Of all 192 clustered pinholes, 162 were being used for image reconstruction. The collimator has an inner diameter of $48 \mathrm{~mm}$, the pinhole centers are placed at a diameter of $64 \mathrm{~mm}$, and the collimator has a wall thickness of $43 \mathrm{~mm}$.

All the pinhole clusters together observe a field of view that extends over the entire collimator tube diameter $(48 \mathrm{~mm})$ and has the shape of an hourglass (21). The average longitudinal length of the collimator field of view is $36 \mathrm{~mm}$. A portion of the field of view, referred to as the central field of view, is sampled by all pinhole clusters simultaneously, and in that portion complete data acquisition is obtained without any translation of the bed. For the clustered-pinhole collimator used here, the central field of view had a diameter of approximately $12 \mathrm{~mm}$ and a longitudinal length of approximately $9 \mathrm{~mm}$.

\section{Image Reconstruction and Calibration}

The scanning focus method was used for data acquisition (22). A pixel-based ordered-subsets expectation maximization algorithm (23) with 16 subsets was used for image reconstruction. The system matrix for the SPECT tracers was obtained using the method described by Van der Have et al. (24). The system matrix for ${ }^{18} \mathrm{~F}$-labeled tracers was calculated using a ray-tracing code (25). Inputs to this code were the precise pinhole and detector positions and detector orientations, determined via point-source measurements (24), whereas pinhole axis orientation and the exact pinhole opening angle were estimated from a detector image of an extended source.

\section{Scatter Correction}

In all experiments, scatter was corrected using a triple-energywindow technique (26). All photopeak windows were set to a width of $15 \%$. For ${ }^{18} \mathrm{~F}$-labeled tracers, background windows of $400-450$ $\mathrm{keV}$ and $550-600 \mathrm{keV}$ were chosen; for ${ }^{99 \mathrm{~m}} \mathrm{Tc}-$ labeled tracers, these windows were set to $100-115 \mathrm{keV}$ and $155-170 \mathrm{keV}$; and for ${ }^{123} \mathrm{I}-$ labeled tracers, background windows were set to $105-120 \mathrm{keV}$ and 260-275 keV.

\section{Evaluation of System Characteristics}

Resolution for SPECT and PET Tracers. To test the resolution of VECTor for the most common PET and SPECT isotopes $\left({ }^{18} \mathrm{~F}\right.$ and ${ }^{99 \mathrm{~m}} \mathrm{Tc}$ ) in a simultaneous acquisition, a miniature resolution phantom (Jaszczak phantom; Vanderwilt Techniques) with 6 sectors was manufactured. Each sector contains a set of equally sized capillaries with diameters of $0.85,0.8,0.75,0.55,0.5$, and $0.45 \mathrm{~mm}$ (Fig. 2). The distance between capillary centers was equal to twice the capillary diameter. To test if the presence of a PET tracer influenced the resolution of SPECT images, we covered a wide range of SPECTto-PET tracer concentration ratios in the resolution phantom scan 


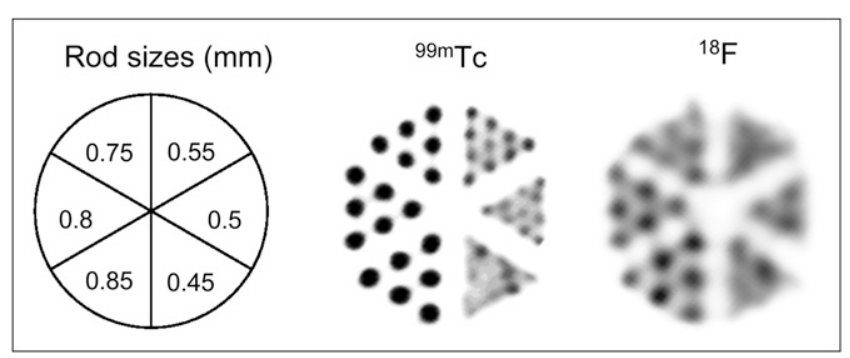

FIGURE 2. Simultaneously acquired images of SPECT and PET tracers in phantom with 6 segments with capillary diameters of 0.85 , $0.80,0.75,0.55,0.50$, and $0.45 \mathrm{~mm}$. At start of this scan, capillaries contained $14 \mathrm{MBq}$ of ${ }^{18} \mathrm{~F}$ solution and $8.3 \mathrm{MBq}$ of ${ }^{99 \mathrm{~m} T c}$ solution. Phantom was imaged for $60 \mathrm{~min}$. All images shown had slice thickness of $3.2 \mathrm{~mm}$.

by performing a scan over more than 10 half-lives of ${ }^{18} \mathrm{~F}$ (20 h of total scanning time), with the acquired data split into time frames of $1 \mathrm{~h}$. At the beginning of the scan series, capillaries contained 29.8 $\mathrm{MBq}$ of ${ }^{18} \mathrm{~F}$ solution mixed with $10.4 \mathrm{MBq}$ of ${ }^{99 \mathrm{~m}} \mathrm{Tc}$ solution. In this paper, we show SPECT/PET images of the scan starting at $2 \mathrm{~h}$ (14 MBq of ${ }^{18} \mathrm{~F}$ and $8.3 \mathrm{MBq}$ of ${ }^{99 \mathrm{~m} T c}$ solution); other images from this scan series are provided in the supplemental data (available online at http://jnm.snmjournals.org).

Phantom with Cylindric Compartments. Some amount of PET tracer may be expected to "leak" into the SPECT images, because the $511-\mathrm{keV}$ annihilation photons produced by the PET tracer can down-scatter into the SPECT photopeak. This effect can lead to overestimation of the amount of SPECT tracer in a certain volume of interest where PET tracer is also present. To test if such an effect occurred, we used a cylindric phantom ( $25 \mathrm{~mm}$ in diameter, $25 \mathrm{~mm}$ in length) containing 2 smaller cylindric compartments (7 $\mathrm{mm}$ in diameter, $12 \mathrm{~mm}$ in length) (Supplemental Fig. 2A). The large cylindric volume was filled with water. The left small cylinder was filled with a mix of approximately $20 \mathrm{MBq}$ of ${ }^{99 \mathrm{~m}} \mathrm{Tc}$ and $50 \mathrm{MBq}$ of ${ }^{18} \mathrm{~F}$ solution, and the right one was filled with approximately $19 \mathrm{MBq}$ of ${ }^{99 \mathrm{~m}} \mathrm{Tc}$ solution. By comparing estimated amounts of SPECT tracers in the left and right compartments over time, we investigated if the PET tracer in the left compartment hindered accurate estimation of the amount of SPECT tracer in that same compartment. In reality, this SPECT-to-SPECT tracer ratio remains constant during the scan, but if the presence of PET tracer influences quantification of SPECT tracer in the left compartment this ratio is expected to become time-dependent. (Because the PET tracer decays much more rapidly than the SPECT tracer, its effect on SPECT quantification will also diminish over time.) To this end, we scanned for $14 \mathrm{~h}$ with data split into 60-min scans. Uptake of tracer in each compartment was determined by drawing a cylinder around the compartments with a diameter of 10 $\mathrm{mm}$, larger than the cylinders themselves, to avoid partial-volume effects. SPECT and PET images were scaled to the same relative intensities using calibration factors for ${ }^{99 \mathrm{~m}} \mathrm{Tc}$ and ${ }^{18} \mathrm{~F}$ that were determined by scanning a small source filled with a known quantity of ${ }^{99 \mathrm{~m}} \mathrm{Tc}$ or ${ }^{18} \mathrm{~F}$ and then applying attenuation correction (27).

Sensitivity. A small source was made by enclosing $6 \mu \mathrm{L}$ of ${ }^{18} \mathrm{~F}$ or ${ }^{99 \mathrm{~m}} \mathrm{Tc}$ solution in a cylinder with a diameter of $2 \mathrm{~mm}$. Sensitivity profiles were acquired by moving this source over 3 mutually perpendicular axes that crossed in the center of the collimator. The sensitivity was defined as the ratio of the number of detected $\gamma$-photons to the number of (positron or $\gamma$ ) emissions. Sensitivity was determined for photopeak windows with widths of $30 \%, 25 \%$, $20 \%$, and $15 \%$.

\section{Animal Studies}

Animal studies were performed in accordance with the Dutch Law on Animal Experimentation and protocols approved by the Animal Research Committee of the University Medical Center Utrecht.

Mouse Heart Scan. A 29-g C57B16 mouse was anesthetized with isoflurane and then injected intravenously with $130 \mathrm{MBq}$ of ${ }^{99 m}$ Tc-tetrofosmin and $24 \mathrm{MBq}$ of ${ }^{18} \mathrm{~F}-\mathrm{FDG}$. A 60-min SPECT/ PET acquisition was obtained, starting $10 \mathrm{~min}$ after injection.

Mouse Brain Scan. A 23-g C57B16 mouse, which had been kept fasting for $12 \mathrm{~h}$, was injected intraperitoneally with $40 \mathrm{MBq}$ of ${ }^{18} \mathrm{~F}-\mathrm{FDG}$ and $30 \mathrm{MBq}$ of ${ }^{123} \mathrm{I}-\mathrm{N}$ - $\omega$-fluoropropyl-2 $\beta$-carbomethoxy$3 \beta$-(4-iodophenyl)nortropane ( ${ }^{123}$ I-FP-CIT). The mouse was anesthetized with isoflurane $45 \mathrm{~min}$ after radionuclide injection. A 60-min SPECT/PET acquisition was obtained, starting $105 \mathrm{~min}$ after injection.

Mouse Bone Scans. A 33-g C57Bl6 mouse was anesthetized with isoflurane and injected intravenously with $220 \mathrm{MBq}$ of $99 \mathrm{mTc}-$

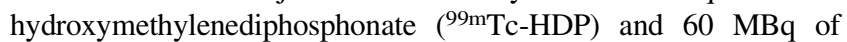
${ }^{18}$ F-fluoride. A 60-min whole-body SPECT/PET acquisition was obtained, starting $30 \mathrm{~min}$ after injection.

A 27-g C57B16 mouse was anesthetized with isoflurane and injected intravenously with $130 \mathrm{MBq}$ of ${ }^{99 \mathrm{~m}} \mathrm{Tc}-\mathrm{HDP}$ and $85 \mathrm{MBq}$ of ${ }^{18}$ F-fluoride. A 30-min SPECT/PET acquisition of the lumbar spine and pelvis was obtained, starting $60 \mathrm{~min}$ after injection.

\section{RESULTS}

\section{Evaluation of System Characteristics}

Resolution for SPECT and PET Tracers. SPECT and PET images of the resolution phantom filled with a mix of ${ }^{99 \mathrm{~m}} \mathrm{Tc}$ and ${ }^{18} \mathrm{~F}$ solution (SPECT-to-PET concentration ratio, $\sim 1: 1.7$ ) are shown in Figure 2. Rods of $0.5 \mathrm{~mm}$ can be distinguished in the ${ }^{99 \mathrm{~m}} \mathrm{Tc}$ image, and $0.8-\mathrm{mm}$ rods are still clearly visible for ${ }^{18} \mathrm{~F}$.

Supplemental Figure 1 shows SPECT images of the same phantom with different SPECT-to-PET tracer concentration ratios. Even when the concentration of the PET tracer exceeded that of the SPECT tracer by a factor of 2.9, 0.5$\mathrm{mm}$ rods were still visible in the SPECT images, demonstrating that the resolution of SPECT images was hardly affected by the simultaneous presence of a PET tracer. Such a degrading effect might be expected to occur because of down-scatter of PET annihilation photons into the SPECT photopeak.

Phantom with Cylindric Compartments. The ratio of the amount of SPECT tracer $\left({ }^{99 \mathrm{~m}} \mathrm{Tc}\right)$ in the left and right compartments of the water-filled mouse-sized phantom (Supplemental Fig. 2A) is shown in Supplemental Figure 2B as a function of the relative amount of PET tracer $\left({ }^{18} \mathrm{~F}\right)$ that was also present in the left compartment. Because all data points result from multiple time frames of a single scan, the true SPECT-to-SPECT tracer ratio is constant. However, leakage of PET tracer distribution into the SPECT images would affect this ratio, an effect that is expected to decrease over time because of the relatively rapid decay of the PET tracer. The experimental results demonstrated that, even 
though the SPECT-to-PET tracer concentration ratio in the left compartment varied widely during the scan (from 1:2.5 to $12: 1$ ), the SPECT-to-SPECT tracer ratio of the 2 cylinders did not vary much. In fact, this ratio differed by only $3.8 \%$ between the first and last time points of the scan. Furthermore, SPECT image slices and profiles showed no activity in the water-filled area (Supplemental Fig. 2B).

Sensitivity. The peak sensitivity of the system for ${ }^{99 \mathrm{~m}} \mathrm{Tc}$ and ${ }^{18} \mathrm{~F}$ for different photopeak window widths is provided in Table 1. For a photopeak window width of $30 \%,{ }^{99 \mathrm{~m}} \mathrm{Tc}$ and ${ }^{18} \mathrm{~F}$ peak sensitivities were $0.25 \%$ and $0.31 \%$, respectively. The system sensitivity differed only slightly for photopeak windows of 25\%, 20\%, and 15\%. In Supplemental Figure 3, sensitivity profiles are provided.

Taken together, these system tests demonstrated that PET and SPECT tracers could be imaged simultaneously with excellent resolutions and minimal cross-talk.

\section{Simultaneous SPECT/PET Images in Living Mice}

To determine whether it would be possible to obtain highly detailed, simultaneous SPECT and PET images of complex biologic systems in mice, we acquired in vivo heart, brain, and bone images with SPECT and PET tracer molecules. All the tracer combinations we tested were chosen with specific biologic applications in mind-applications in which a direct correlation of multiple processes is of importance. Naturally, these combinations represent only a small subset of the imaging studies that clustered-pinhole SPECT/PET now makes possible.

Mouse Heart Scan. First, we performed a dynamic (4dimensional) heart scan on a mouse using a combination of ${ }^{99 m}$ Tc-tetrofosmin (a clinically used SPECT myocardial perfusion tracer) and ${ }^{18}$ F-FDG (used clinically to distinguish between dysfunctional/hibernating but viable myocardial tissue and scar tissue). This SPECT/PET tracer combination is of great practical interest in experimental cardiology, because it might be used to accurately distinguish between viable and scarred myocardium (28). Three perpendicular slices through the mouse heart are shown in Figure 3. These images were averaged over the cardiac cycle. Supplemental Video 1 (4-dimensional) of the beating mouse heart was reconstructed from the same scan with electrocardiography gating). The SPECT components of the cardiac images show somewhat more detail than do the images of the PET tracer. We attribute this mainly to the degree of $\gamma$-photon penetration through the pinhole edges. Furthermore, because

TABLE 1

Measured Peak Sensitivity for ${ }^{99 m T c}$ and ${ }^{18} \mathrm{~F}$

\begin{tabular}{llc}
\hline & \multicolumn{2}{c}{ Sensitivity } \\
\cline { 2 - 3 } Photopeak window width & $99 \mathrm{mTc}$ & $18 \mathrm{~F}$ \\
\hline $30 \%$ & $0.25 \%$ & $0.31 \%$ \\
$25 \%$ & $0.25 \%$ & $0.30 \%$ \\
$20 \%$ & $0.24 \%$ & $0.29 \%$ \\
$15 \%$ & $0.22 \%$ & $0.28 \%$ \\
\hline
\end{tabular}

${ }^{99 m}$ Tc-tetrofosmin - in contrast to ${ }^{18} \mathrm{~F}-\mathrm{FDG}$ - accumulates in the gallbladder, this organ is visible only in the SPECT images.

Mouse Brain Scan. We also acquired simultaneous SPECT/PET brain images, combining a dopamine transporter ligand ( $\left.{ }^{123} \mathrm{I}-\mathrm{FP}-\mathrm{CIT}\right)$ and a glucose analog $\left({ }^{18} \mathrm{~F}-\mathrm{FDG}\right)$, both merged with a CT scan (Fig. 4). Specific binding of ${ }^{123}$ I-FP-CIT to dopamine transporters can be resolved in subregions of the brain (e.g., the striatum and the olfactory tubercle), whereas uptake in the Harderian glands may reflect nonspecific uptake. The images obtained for ${ }^{18} \mathrm{~F}$ FDG showed an overall distribution with high concentrations in the olfactory bulbs and the cortical, thalamic, and midbrain areas. Earlier, we imaged the effects of cocaine (a dopamine transporter blocker) on the temporal characteristics of striatal ${ }^{123} \mathrm{I}-\mathrm{FP}-\mathrm{CIT}$ binding in the mouse brain (16), whereas ${ }^{18} \mathrm{~F}$ FDG has been used as a brain activity marker (29). Imaging this mix of SPECT and PET tracer molecules combined with the administration of a drug of abuse (e.g., cocaine) may create opportunities to directly relate molecular and functional processes in the brain by relating the binding location of the drug (revealed in the case of cocaine by displacement of ${ }^{123}$ I-FP-CIT) to metabolic activity changes (revealed by $\left.{ }^{18} \mathrm{~F}-\mathrm{FDG}\right)$ in the brain.

Mouse Bone Scan. Finally, to illustrate a typical example of how SPECT and PET tracers suitable for a similar imaging task can be compared one-to-one under exactly the same biologic circumstances, we performed parallel skeletal SPECT/PET with the bone tracers ${ }^{18} \mathrm{~F}$-fluoride and ${ }^{99 \mathrm{~m} T c-}$ HDP. Figure 5 shows maximum-intensity projections (MIPs) from a simultaneously acquired whole-body bone scan. Figure 6 shows MIPs from a second bone scan with the same tracer combination in which we focused on the lumbar spine and pelvis. In these focused images, uptake of ${ }^{99 m} \mathrm{Tc}-\mathrm{HDP}$ and ${ }^{18} \mathrm{~F}$-fluoride in tiny parts of the individual processes and other tiny vertebral parts is visualized. Like the cardiac image in Figure 3, the images of the SPECT tracer reveal a bit more detail than do the PET components. Rotating MIPs from the same bone scans are available as Supplemental Videos 2 and 3.

\section{DISCUSSION}

We have shown that SPECT images are hardly degraded by the simultaneous presence of a PET tracer-even when the activity concentration of the PET tracer exceeds that of the SPECT tracer by up to a factor of 2.5 (Supplemental Figs. 1 and 2). Typically, activities used in biologic SPECT experiments are higher than those in PET experiments (30). To confirm this, we performed a search for the 20 most recent papers on mouse SPECT and on mouse PET published in The Journal of Nuclear Medicine (search terms mice SPECT or mice PET). These papers report average injected activities of $52 \mathrm{MBq}$ and $8.1 \mathrm{MBq}$ for mouse SPECT and mouse PET, respectively. As a result, we predict that in most biologic experiments, spill-over from the PET tracer into the SPECT images will be negligible, 


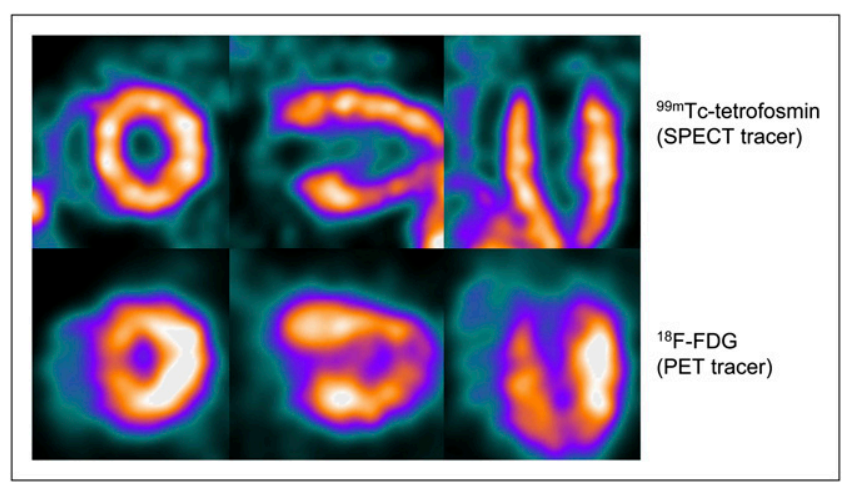

FIGURE 3. Simultaneous SPECT/PET cardiac scan. We imaged

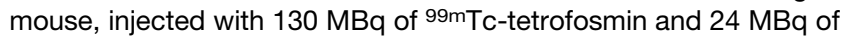
${ }^{18} \mathrm{~F}-\mathrm{FDG}$, for $60 \mathrm{~min}$. Here, we show 3 mutually perpendicular slices through mouse's heart. Images represent average over cardiac cycle. Supplemental Video 1, of beating heart, was reconstructed from same scan.

allowing for significant flexibility in the use of tracers and consequently a greater versatility in their application to biologic systems.

We were able to obtain high-resolution PET images despite the relatively low sensitivity (peak sensitivity of $0.31 \%$ ) of pinhole PET compared with coincidence PET (peak sensitivities typically in the $1 \%-10 \%$ range). The level of detail visible in the images shows that sensitivity alone is insufficient as a predictor of image quality. In fact, it is the combination of sensitivity and the accuracy with which the direction of a detected $\gamma$-photon is known that determines how much image detail can be obtained.

In this paper we have presented focused scans, restricted to a specific organ or part of the mouse, as well as whole-body images. Because all clusters in the VECTor collimator focus on a central scan volume that is smaller than a mouse, focusing on a restricted volume of interest can increase the number of detected counts from that region, resulting in improved noise-resolution trade-offs (21). The benefits of focusing are well illustrated by the mouse bone scans in Figures 5 and 6; the visibility of small details is enhanced in the focused scan, compared with the whole-body scan. For this scan, simulations indicated that focusing increased the average sensitivity over the volume of interest by a factor of 5 .

We obtained the images presented in this paper by placing the novel collimator into an existing SPECT system. This system has $\gamma$-detectors that are optimized for imaging SPECT tracers. The crystals have a thickness of $9 \mathrm{~mm}$, which means that only about $10 \%$ of the $511-\mathrm{keV} \gamma$-photons that reach the detector are detected in the photopeak (25). The implication is that there is ample room for improving the sensitivity of the system. Increases of up to a factor of 8 might be achieved simply by, for example, using an 80-mm-thick NaI crystal. We estimated that down-scatter of 511-keV $\gamma$-photons entering the NaI crystal and detected at $140 \mathrm{keV}$ would then decrease from $0.9 \%$ to $0.6 \%$, thus reducing contamination of SPECT images. If, in addition, larger pinholes (e.g., 1.0 instead of $0.7 \mathrm{~mm}$ ) were to be used, peak sensitivities similar to those in coincidence small-animal PET could readily be obtained. Future research on pinhole SPECT/PET will certainly include the optimization of detectors and geometry (e.g., size and shape of pinholes and number of pinholes per cluster).

Clustered-pinhole SPECT/PET has not been designed with a single imaging application in mind but rather as an enabling technology for use in a variety of research areas in which a direct correlation of multiple biologic functions will lead to enhanced insights. With simultaneous SPECT/ PET, we enable a whole new range of applications in biomedical research, since 2 tremendously large and important classes of tracers can now be combined for the first time.

In cardiovascular research, a SPECT/PET tracer combination has already attracted considerable attention over the last 2 decades. It involves the use of ${ }^{18} \mathrm{~F}$-FDG together with a blood-flow tracer (such as ${ }^{99 \mathrm{~m}} \mathrm{Tc}$-tetrofosmin or ${ }^{201} \mathrm{Tl}$ ). Such a tracer combination permits the assessment of both myocardial glucose utilization and flow in a single study (28). However, until now, the lack of suitable technology has hampered its high-resolution application. Small-animal models of disease play an important role in the optimization of imaging procedures and in the development of tracers and therapies. The high-resolution mouse heart images presented in Figure 3 show that the proposed cardiovascular tracer combinations can now be applied in a preclinical setting.

High-resolution SPECT/PET may also play a significant role in simultaneous and possibly dynamic imaging of different brain processes, such as metabolic activity in defined brain regions together with the functioning of the neurotransmitter system (Fig. 4)—or, alternatively, in monitoring various aspects of the neurotransmitter system simultaneously.

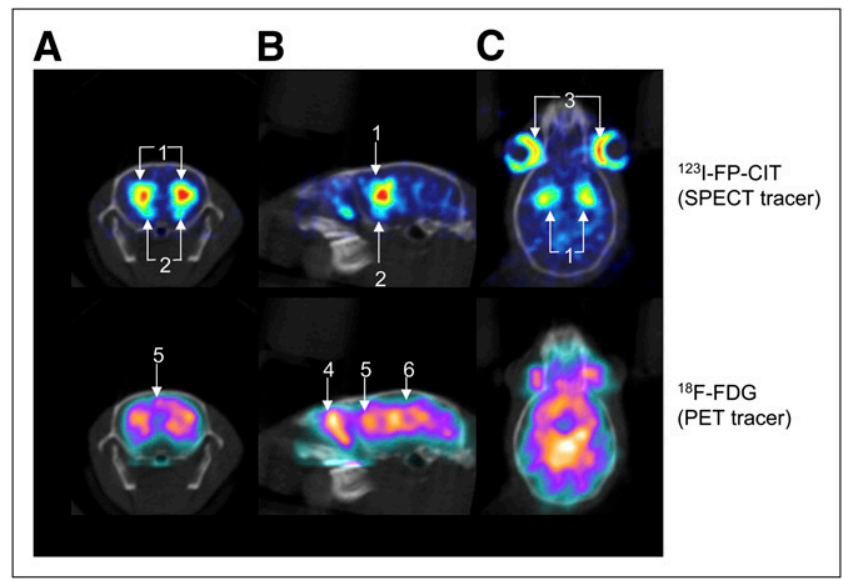

FIGURE 4. Simultaneously acquired SPECT/PET mouse brain images (color) overlayed on CT (gray). Coronal (A), sagittal (B), and horizontal $(\mathrm{C})$ slices at identical levels are shown. Mouse was injected with $30 \mathrm{MBq}$ of ${ }^{123} \mathrm{I}-\mathrm{FP}-\mathrm{ClT}$ and $40 \mathrm{MBq}$ of ${ }^{18} \mathrm{~F}-\mathrm{FDG}$ and imaged for 60 min starting $105 \mathrm{~min}$ after injection. Uptake of ${ }^{123}$ IFP-CIT (SPECT, top) in small brain structures such as striatum (1), olfactory tubercle (2), and Harderian glands (3) can be resolved. ${ }^{18} \mathrm{~F}$ FDG (PET scan, bottom) is seen in multiple structures, including olfactory bulbs (4), cerebral cortex (5), and thalamic and midbrain areas (6). 


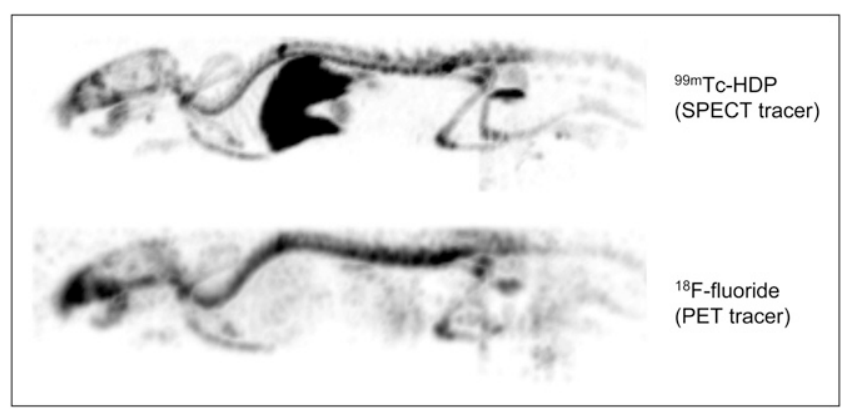

FIGURE 5. Example of simultaneous SPECT/PET scan obtained with 2 tracers that target same biologic function. Images represent MIPs of mouse injected with 2 bone tracers, $220 \mathrm{MBq}$ of ${ }^{99 m T c}$-HDP (top) and $60 \mathrm{MBq}$ of ${ }^{18} \mathrm{~F}$-fluoride (bottom), and scanned for $60 \mathrm{~min}$. Supplemental Video 2 shows rotating MIPs.

Specifically, many research groups are currently investigating the effect of an intervention (e.g., amphetamine or cocaine administration) on metabolic brain activity (monitored with ${ }^{18} \mathrm{~F}-\mathrm{FDG}$ ) or on the neurotransmitter system (imaged with SPECT tracers such as IBZM, FP-CIT, or TRODAT). Because the biologic system does not react in the same way to repeated interventions (31) (an effect called sensitization), these 2 effects can be directly correlated only through simultaneous SPECT/PET. Furthermore, in research related to Parkinson disease, the possibility of using simultaneous SPECT/ PET to image multiple sites or processes of interest-thereby enabling a direct investigation of interactions between different processes-has already attracted interest (32).

In tumor imaging, ${ }^{18} \mathrm{~F}-\mathrm{FDG}$ is widely used as a marker of tumor response, whereas in other studies, long-living SPECT tracers such as ${ }^{111} \mathrm{In}$ or ${ }^{125} \mathrm{I}$ are attached to antibodies used for anticancer therapy to determine their distribution. With simultaneous SPECT/PET, these different aspects of tumor imaging can now be combined in a single longitudinal study. In this way, a repeated read-out of therapy effects can be directly related to the amount and distribution of SPECTlabeled pharmaceutical within the tumor.

In addition, simultaneous SPECT/PET can be used in tracer research to compare a SPECT and a PET tracer that target the same biologic function (Figs. 5 and 6). In a simultaneous SPECT/PET scan, the targeting properties of a molecule labeled with a heavy metal-such as is commonly used for SPECT tracers-could be compared directly with the behavior of an identical molecule labeled with a small positronemitting radioactive atom. Such a comparison, at the same time and at exactly the same position in a single animal, rules out the effects of varying physiology and spatiotemporal misregistration-effects that today severely complicate tracer comparisons. This, in turn, can aid in the modification of a PET tracer into a SPECT tracer. The modification of PET tracers into SPECT tracers is of practical interest because of the wider availability of SPECT and may also be necessary when a process needs to be monitored for a long time-something prohibited by the relatively short lifetimes of most PET labels.
Previously, we showed that mouse organs and entire bodies can be imaged with half-minute time frames using high-resolution focused multipinhole SPECT $(16,17)$. Because VECTor has a better sensitivity than these SPECT systems (both for SPECT and PET tracers), we expect that similar time resolutions will be feasible, allowing, for example, tracer kinetic modeling in simultaneous SPECT/PET.

The clustered-pinhole technology presented here may also lend itself to other novel applications in biomedical imaging. For example, suppose that the clusters were positioned closer to one other. Such a set-up would result in a severe overlap of pinhole projections-which in most situations would be undesirable $(33,34)$. However, by adding small shutters that block a subset of the pinholes within each cluster, one could create imaging devices capable of focusing rapidly and flexibly and that could quickly be adapted to specific imaging needs-for example, for highly specific imaging of regions of the brain containing certain receptors or plaques, or for focusing on a tumor. Adaptive imaging of radionuclides has recently drawn much attention (35), and many new developments can be expected in this emerging area of research.

\section{CONCLUSION}

We have shown that collimated simultaneous imaging of SPECT and PET tracers with submillimeter image resolutions is possible even when detectors are used that are suboptimal for detecting $511-\mathrm{keV} \gamma$-photons resulting from PET tracers. From the large number of specific SPECT/PET tracer combinations that are enabled by our new technol-

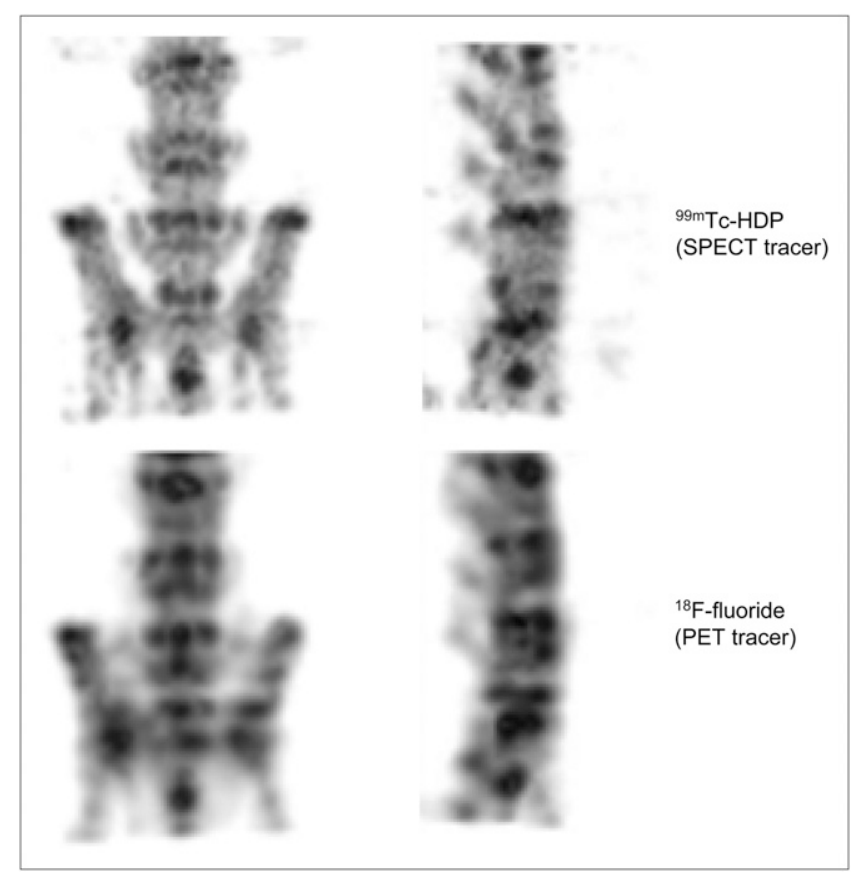

FIGURE 6. Example of simultaneous SPECT/PET bone scan of lumbar spine and pelvis. MIPs of mouse injected with $130 \mathrm{MBq}$ of ${ }^{99 \mathrm{~m} T c-H D P}$ (top) and $85 \mathrm{MBq}$ of ${ }^{18} \mathrm{~F}$-fluoride (bottom) and scanned for $30 \mathrm{~min}$ are shown. Supplemental Video 3 shows rotating MIPs. 
ogy, we have selected 3 prominent examples, and we have demonstrated our ability to simultaneously acquire separate images of these probes with excellent resolutions. This evidence leads us to believe that clustered-pinhole SPECT/ PET may create unique imaging opportunities in a large number of biologic research applications.

\section{DISCLOSURE STATEMENT}

The costs of publication of this article were defrayed in part by the payment of page charges. Therefore, and solely to indicate this fact, this article is hereby marked "advertisement" in accordance with 18 USC section 1734.

\section{ACKNOWLEDGMENTS}

We thank René Gommers and René den Oudsten for their technical assistance in the construction of the collimator. This research was cofunded by grant PID06015, forming part of the "Pieken in de Delta Zuidvleugel" program of the Netherlands Ministry of Economic Affairs and the Province of Zuid-Holland. No other potential conflict of interest relevant to this article was reported.

\section{REFERENCES}

1. Rowland DJ, Cherry SR. Small-animal preclinical nuclear medicine instrumentation and methodology. Semin Nucl Med. 2008;38:209-222.

2. Weissleder R, Ross BD, Rehemtulla A, Gambhir SS. Molecular Imaging: Principles and Practice. Shelton, CT: People's Medical Publishing HouseUSA; 2010:844-1178.

3. Jaszczak RJ, Li JY, Wang HL, Zalutsky MR, Coleman RE. Pinhole collimation for ultra-high-resolution, small-field-of-view spect. Phys Med Biol. 1994;39: 425-437.

4. Ishizu K, Mukai T, Yonekura Y, et al. Ultra-high-resolution spect system using 4 pinhole collimators for small animal studies. J Nucl Med. 1995;36: 2282-2287.

5. Liu Z, Kastis GA, Stevenson GD, et al. Quantitative analysis of acute myocardial infarct in rat hearts with ischemia-reperfusion using a high-resolution stationary SPECT system. J Nucl Med. 2002;43:933-939.

6. Furenlid LR, Wilson DW, Chen YC, et al. FastSPECT II: a second-generation highresolution dynamic SPECT imager. IEEE Trans Nucl Sci. 2004;51:631-635.

7. Metzler SD, Jaszczak RJ, Patil NH, Vemulapalli S, Akabani G, Chin BB. Molecular imaging of small animals with a triple-head SPECT system using pinhole collimation. IEEE Trans Med Imaging. 2005;24:853-862.

8. van der Have F, Vastenhouw B, Ramakers RM, et al. U-SPECT-II: an ultrahigh-resolution device for molecular small-animal imaging. J Nucl Med. 2009; 50:599-605.

9. Beekman F, van der Have F. The pinhole: gateway to ultra-high-resolution three-dimensional radionuclide imaging. Eur J Nucl Med Mol Imaging. 2007;34:151-161.

10. Jeavons AP, Chandler RA, Dettmar CAR. A 3D HIDAC-PET camera with submillimetre resolution for imaging small animals. IEEE Trans Nucl Sci. 1999; 46:468-473.

11. Tai YC, Chatziioannou AF, Yang YF, et al. MicroPET II: design, development and initial performance of an improved microPET scanner for small-animal imaging. Phys Med Biol. 2003;48:1519-1537.
12. Yang Y, Tai YC, Siegel S, et al. Optimization and performance evaluation of the microPET II scanner for in vivo small-animal imaging. Phys Med Biol. 2004; 49:2527-2545.

13. Rouze NC, Schmand M, Siegel S, Hutchins GD. Design of a small animal PET imaging system with 1 microliter volume resolution. IEEE Trans Nucl Sci. 2004; 51:757-763.

14. Miyaoka RS, Janes ML, Lee K, Park B, Kinahan PE, Lewellen TK. Development of a single detector ring micro crystal element scanner: QuickPET II. Mol Imaging. 2005;4:117-127.

15. Tai YC, Ruangma A, Rowland D, et al. Performance evaluation of the microPET focus: a third-generation microPET scanner dedicated to animal imaging. $J$ Nucl Med. 2005;46:455-463.

16. Vastenhouw B, van der Have F, van der Linden AJ, et al. Movies of dopamine transporter occupancy with ultra-high resolution focusing pinhole SPECT. Mol Psychiatry. 2007;12:984-987.

17. Vaissier PEB, Goorden MC, Vastenhouw B, Van der Have F, Ramakers RM, Beekman FJ. Fast spiral SPECT with stationary $\gamma$-cameras and focusing pinholes. J Nucl Med. 2012;53:1292-1299.

18. Goorden MC, Beekman FJ. High-resolution tomography of positron emitters with clustered pinhole SPECT. Phys Med Biol. 2010;55:1265-1277.

19. Beekman FJ, inventor; MILabs BV, assignee. Focused pinhole gamma detection device. U.S. patent 8,067,741. November 29, 2011. European patent application EP2073039.

20. Bom V, Goorden M, Beekman F. Comparison of pinhole collimator materials based on sensitivity equivalence. Phys Med Biol. 2011;56:3199-3214.

21. Branderhorst W, Vastenhouw B, van der Have F, Blezer ELA, Bleeker WK, Beekman FJ. Targeted multi-pinhole SPECT. Eur J Nucl Med Mol Imaging. 2011;38:552-561.

22. Vastenhouw B, Beekman F. Submillimeter total-body murine imaging with USPECT-I. J Nucl Med. 2007;48:487-493.

23. Branderhorst W, Vastenhouw B, Beekman FJ. Pixel-based subsets for rapid multi-pinhole SPECT reconstruction. Phys Med Biol. 2010;55:2023-2034.

24. van der Have F, Vastenhouw B, Rentmeester M, Beekman FJ. System calibration and statistical image reconstruction for ultra-high resolution stationary pinhole SPECT. IEEE Trans Med Imaging. 2008;27:960-971.

25. Goorden MC, van der Have F, Kreuger R, Beekman FJ. An efficient simulator for pinhole imaging of PET isotopes. Phys Med Biol. 2011;56:1617-1634.

26. Ogawa K, Harata Y, Ichihara T, Kubo A, Hashimoto S. A practical method for position-dependent Compton-scatter correction in single photon-emission ct. IEEE Trans Med Imaging. 1991;10:408-412.

27. Wu C, van der Have F, Vastenhouw B, Dierckx RAJO, Paans AMJ, Beekman FJ. Absolute quantitative total-body small-animal SPECT with focusing pinholes. Eur J Nucl Med Mol Imaging. 2010;37:2127-2135.

28. Delbeke D, Videlefsky S, Patton JA, et al. Rest myocardial perfusion/metabolism imaging using simultaneous dual-isotope acquisition spect with technetium99m-MIBI/fluorine-18-FDG. J Nucl Med. 1995;36:2110-2119.

29. Reiman EM, Uecker A, Gonzalez-Lima F, et al. Tracking Alzheimer's disease in transgenic mice using fluorodeoxyglucose autoradiography. Neuroreport. 2000; 11:987-991.

30. Funk T, Sun MS, Hasegawa BH. Radiation dose estimate in small animal SPECT and PET. Med Phys. 2004;31:2680-2686.

31. O'Daly OG, Joyce D, Stephan KE, Murray RM, Shergill SS. Functional magnetic resonance imaging investigation of the amphetamine sensitization model of schizophrenia in healthy male volunteers. Arch Gen Psychiatry. 2011;68:545-554.

32. Stoessl AJ, Martin WRW, McKeown M, Sossi V. Advances in imaging in Parkinson's disease. Lancet Neurol. 2011;10:987-1001.

33. Vunckx K, Suetens P, Nuyts J. Effect of overlapping projections on reconstruction image quality in multipinhole SPECT. IEEE Trans Med Imaging. 2008;27:972-983.

34. Mok GS, Tsui BM, Beekman FJ. The effects of object activity distribution on multiplexing multi-pinhole SPECT. Phys Med Biol. 2011;56:2635-2650.

35. Barrett HH, Furenlid LR, Freed M, et al. Adaptive SPECT. IEEE Trans Med Imaging. 2008;27:775-788. 